\title{
Implantatassoziierte Pseudarthrosen - wie kann man sie vermeiden?
}

Volker Bühren, Simon Hackl, Peter Augat

\author{
Pseudarthrosen bilden zusammen mit Fehlstellungen und Infekten die drei großen \\ Ursachen für Störungen der Knochenbruchheilung. Alle drei Ursachen stehen über \\ verschiedenste Mechanismen miteinander in Verbindung. Eine wesentliche Rolle \\ spielen Planung, Auswahl und Einbringtechnik der Implantate für die Osteosynthese. \\ In diesem Kapitel sollen typische vom Implantat beeinflusste Faktoren mit Fraktur- \\ heilungsstörung beschrieben werden, mit besonderem Augenmerk auf eine \\ mögliche Prävention der Heilungsstörung.
}

\section{Pseudarthrose: Patient, Chirurg und Implantat}

Die Liste der Faktoren, die für die Entstehung einer Pseudarthrose ursächlich sein können, ist lang. Frakturform und Weichteilschaden sind quasi vorgegeben und haben wesentlichen Einfluss auf Stabilität und Blutversorgung und damit auf die Heilungspotenz des verletzten Knochens. Ebenfalls weitgehend vorbestehend und nur bedingt beeinflussbar sind die systemischen Vorbedingungen des verletzten Menschen. Zu nennen sind Alter, Vorerkrankung, Medikation, Genussgifte und Trainingszustand. Alle vorgenannten Faktoren haben einen bedeutenden Einfluss auf die Wahl und Ausführung der Osteosynthese und führen nicht selten auch zur Entscheidung, eine nicht operative, konservative Frakturbehandlung durchzuführen. Einzubeziehen sind auch die absehbaren funktionellen Ansprüche und Limitierungen des Patienten. Ist eine Teilbelastung realistisch? Bestehen Einschränkungen der mentalen Compliance, die ein Verstehen und Befolgten der Anweisungen für die postoperative Phase unwahrscheinlich erscheinen lassen?

Wahl des Osteosyntheseverfahrens, operativer Zugang und Ausführung der Instrumentierung sind Faktoren, die der Chirurg oder die Chirurgin wesentlich beeinflussen kann und die damit als dynamische Einflussgrößen den Erfolg (z. B. eine ungestörte Bruchheilung) oder den Misserfolg (z. B. eine Pseudarthrosenentstehung) letztendlich bestimmen. Studien zur gestörten Knochenbruchheilung zeigen durchgehend, dass von den Parametern lokale Verletzung, systemischer Gesamtzustand und operative Behandlung die Durchführung der Osteosynthese wesentlich oder zumindest in der Rolle eines erheblichen Co-Faktors für die Entstehung der Pseudarthrose verant- wortlich ist [1]. Nach aktueller Rechtsprechung und unter dem Ziel einer zeitgemäßen Arzt-Patienten-Interaktion folgert daraus zwangsläufig, dass auch der verletzte Patient, wenn immer möglich, sinnvoll in den Entscheidungsprozess zum therapeutischen Verfahren einschließlich der Implantatwahl aktiv und aufgeklärt mit einbezogen werden muss.

\section{Prävention}

Die Wahl der Osteosyntheseverfahren und der Implantate soll nicht automatisch aufgrund der Diagnose oder nur nach Betrachten des Röntgenbildes, sondern unter Gesamtschau des Patienten und nach kritischer Analyse der lokalen Verletzungsschwere, des systemischen Allgemeinzustandes und der eigenen chirurgischen Möglichkeiten getroffen werden.

\section{Biologie und Biomechanik}

Betrachtet man die beiden Hauptfaktoren, die für die Knochenbruchheilung ausschlaggebend sind, nämlich Biologie und Biomechanik, drängen sich auf den ersten Blick die mechanischen Eigenschaften der diversen Implantate und Instrumentierungen in den Vordergrund. Die nähere Analyse führt dann aber zwangsläufig zu der Erkenntnis, dass auch die Biologie der Frakturheilung untrennbar von Implantateigenschaften beeinflusst wird.

Die wesentlichen biologischen Einflussgrößen der Implantate sind die chirurgische Zugangsmorbidität und die Vergrößerung des Infektrisikos durch Einbringen des Implantats als Fremdkörper in den Frakturbereich. Implantatspezifisch ist der direkte Einfluss auf die Durchblutung des Knochens, z. B. durch Aufbohren des Markraumes bei Marknagelung oder durch die Druckschädigung des Periosts bei Verplattungen. Diese vorgenannten 
Überlegungen haben zum Begriff der biologischen Osteosynthese als wesentliches komplikationsvermeidendes Konzept geführt.

Nach neueren Erkenntnissen hat die implantatassoziierte Keimbesiedelung für die Ausbildung einer Frakturheilungsstörung eine weit höhere Bedeutung als bisher angenommen [2]. Lange schon bekannt ist die klinisch eindeutige Infektpseudarthrose, sozusagen der GAU unter den biologisch bedingten Knochenheilungsstörungen. Neuere Untersuchungen belegen, dass darüber hinaus bei vielen Heilungsverzögerungen, aber auch bei einer klinisch ungestört erscheinenden Heilung, unterschwellige Keimbesiedelungen nachweisbar sind [3]. In diesem Licht gewinnt die unterschiedliche Keimaffinität der spezifischen Implantate zusätzliches Gewicht.

Letztendlich gilt, dass die Faktoren Biologie und Biomechanik für jeden individuellen Fall einer Pseudarthrose zu analysieren sind. Nahezu immer liegen Kombinationen von Störfaktoren vor, wie die Achsabweichung mit Instabilität, die Instabilität mit naheliegendem Infektverdacht, der Weichteilschaden mit ungenügender Fixierung usw. [4]. So gesehen hat die Implantatwahl und das damit verbundene operative Vorgehen eine zentrale Bedeutung natürlich für die Mechanik, aber eben auch für den Faktor Biologie in der Frakturbehandlung.

\section{Prävention}

Biologie und Biomechanik stehen für die Knochenbruchheilung in enger Synergie. Bei der Analyse einer Frakturheilungsstörung müssen die Faktoren Instabilität, Fehlstellung, Durchblutungsstörung und Infekt checklistenmäßig abgearbeitet werden, Kombinationen sind dabei die Regel.

\section{Biomechanische Prinzipien: Kompression und Schienung}

Die primäre Aufgabe des Osteosyntheseimplantats ist mechanischer Natur. Das Implantat muss die korrekte Wiedereinrichtung der Fraktur unter Erhalt der physiologischen Lastachse ermöglichen (Reposition) und diese über den Heilungszeitraum erhalten (Retention) [5]. Da Implantate bei ihrer Entwicklung für die Erfüllung dieser beiden Aufgaben spezifiziert werden, kommt es bei ihrem Einsatz auf eine korrekte Anwendung unter Einhaltung der Frakturheilungsprinzipien an, zu nennen sind hier Schienung oder Kompression. Nur bei korrekter Anwendung entsprechend der Spezifizierung kann eine komplikationslose Heilung der Fraktur erwartet werden.

Bei der Kompressionsosteosynthese ist darauf zu achten, dass der Frakturspalt so eng wie möglich zu komprimieren und somit die Fraktur bestmöglich zu stabilisieren ist. Bei der Schienung hingegen werden minimale Fraktur- spalte toleriert, wobei sich eine gewisse Mikrobewegung in diesen Spalten positiv auf die Frakturheilung auswirkt. Hierbei konnte gezeigt werden, dass eine Frakturspaltbewegung in Belastungsrichtung, also Kompression, die Heilung fördert, wohingegen Scherbewegungen und Rotationen die Knochenbildung im Frakturspalt verzögern [6].

Osteosynthesen im Gelenkbereich erfordern i.d.R. eine perfekte Reposition mit einer bis zur Frakturheilung unveränderten Retention und werden deshalb über eine Kompression mit rigider Fixierung der Fragmente durchgeführt. Osteosynthesen im Schaftbereich profitieren in aller Regel von den biologischen Vorteilen der Schienung, wobei die mechanischen Eigenschaften der spezifischen Implantate für jede Fraktursituation berücksichtigt werden müssen.

In der Praxis müssen für eine Frakturversorgung nicht selten die Prinzipien von Kompression und Schienung kombiniert werden, was zu Kompromissen mit Blick auf die Frakturheilung führen kann. Ein typisches Beispiel ist die Versorgung einer Typ-C-Fraktur der proximalen Tibia, wobei die Gelenkfragmente mit einem hochstabilen Implantat wie der LISS-Platte zu fixieren sind, das allerdings für die Schienung der Schaftkomponente der Fraktur zu rigide sein und zur Pseudarthrose führen kann ( $\bullet$ Abb. 1).

\section{Prävention}

Die Prinzipien der rigiden Fixierung unter Kompression für Gelenkfrakturen und der dynamischen Schienung für Schaftfrakturen stehen bei kombinierten gelenknahen Bruchformen häufig in Konkurrenz. Geeignete Präventionsmaßnahmen für Fehlstellungen und Pseudarthrosenbildungen sind engmaschige Kontrollen mit frühzeitig durchzuführenden Sekundäreingriffen wie z.B. Verplattung der Gegenkortikalis und Knochenimplantation.

\section{Gelenkfrakturen}

Intraartikuläre Frakturen sind nach bestmöglicher Reposition der Bruchstücke stabil und damit unter Fragmentkompression zu fixieren. Bereits geringfügige Dehiszenzen können zu Fehlstellungen des Gelenkes und damit zu einer frühen posttraumatischen Arthrose führen. Besonders bei den lasttragenden Gelenken der unteren Extremität ist daher auf eine ausreichende mechanische Stabilität des Implantats zu achten. Dabei kann es hilfreich sein, das Primärimplantat durch zusätzliche Kompressions- oder Stellschrauben zu unterstützten. Nicht zu vernachlässigen ist die Rekonstruktion stabilisierender ligamentärer Strukturen. Ein praxisrelevantes Beispiel ist die Refixierung der Rotatorenmanschette bei proximalen Humerusfrakturen.

Heilungsverzögerungen und Repositionsverluste bei Frakturen im Gelenkbereich sind meist auf unzureichende 

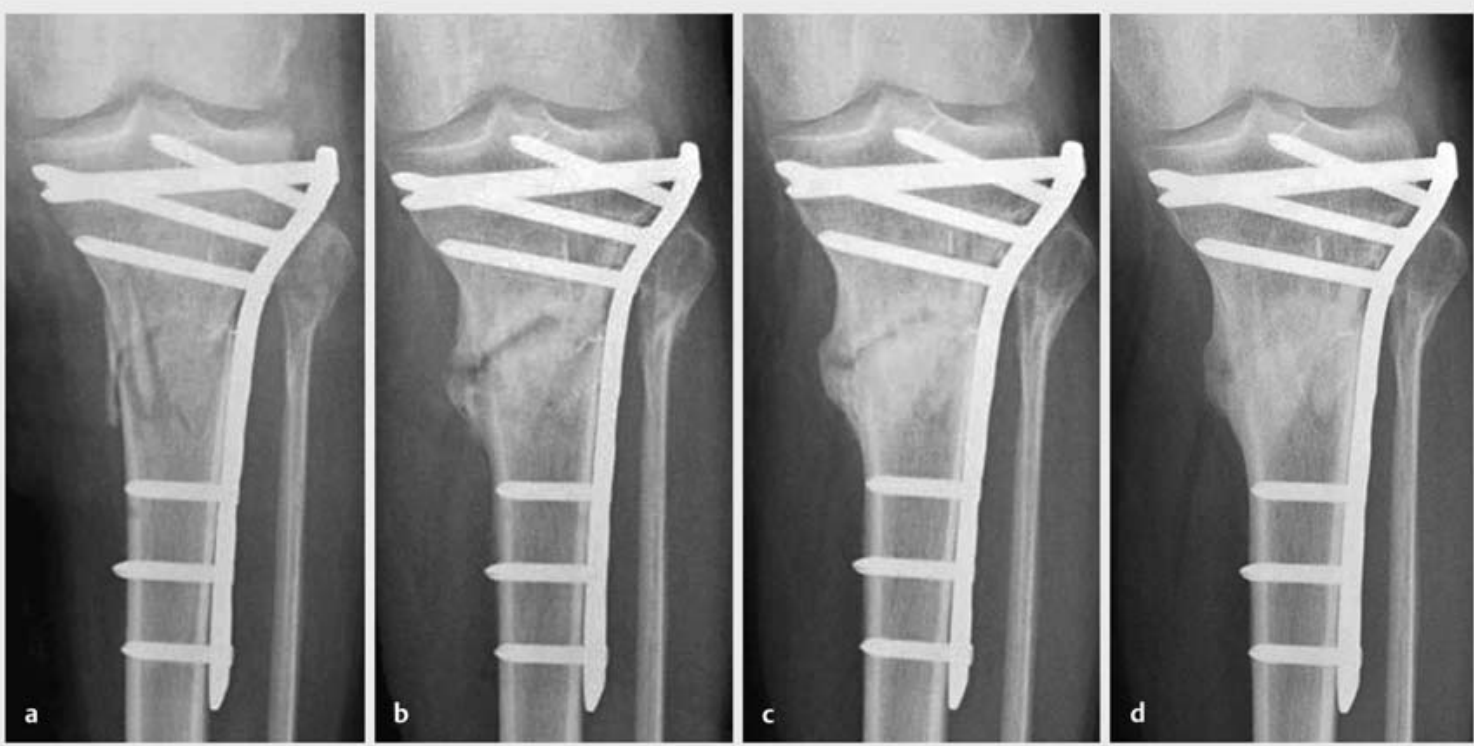

- Abb. 1 59-jährige Frau, proximale Tibiafraktur. a Versorgung mit lateraler LISS-Platte. b Nach 4 Monaten mit verzögerter Bruchheilung. c Nach 10 Monaten mit hypertropher Pseudarthrose bei stabil liegendem Implantat. d Nach 3-maliger Stoßwellentherapie Ausheilung nach 14 Monaten.

Fixierung des Implantats im Knochen zurückzuführen. Entweder wurde primär schon keine ausreichende Stabilität erreicht oder es kommt, so vor allem bei osteoporotischem Knochen, sekundär zu einer Auslockerung oder Durchwandern des Implantats durch den Knochen. Zur Vermeidung sollten Implantate, und hier vor allem die Schrauben, in möglichst kräftigem Knochen zu liegen kommen [7]. So wird für die Fixierung bei hüftgelenknahen Frakturen empfohlen, die Schenkelhalsschraube zentral und möglichst gelenknah im subkortikalen Knochen des Hüftkopfes zu platzieren [8]. Ein weiteres Beispiel bildet die Fixierung proximaler Humerusfrakturen mit einer Platzierung der einzelnen Schrauben im subkortikalen Bereich des Humeruskopfes und biomechanisch sinnvoller Abstützung der medialen Säule durch entsprechend platzierter Schrauben. Bei mangelhafter Knochenqualität kann Knochenzement zur Auffüllung von Defekten oder Augmentation von Schrauben eingesetzt werden.

\section{Prävention}

Der Schlüssel zum Erfolg in der Behandlung artikulärer und gelenknaher Frakturen liegt in der anatomischen Reposition und einer optimalen Platzierung der gelenknahen Schrauben.

\section{Schaftfrakturen}

Bei Frakturen der langen Röhrenknochen ist auf Basis der Frakturform zu entscheiden, ob eine stabile komprimierende Osteosynthese erreicht werden kann. Die Fraktur- reposition kann direkt über eine Zugschraubenosteosynthese oder auch über eine Plattenosteosynthese mit indirekter Kompression des Frakturspaltes durchgeführt werden. Cerclagen können bei Schrägfrakturen für die Einrichtung der Fraktur und das Schließen des Frakturspaltes hilfreich sein. Die Vermeidung eines klaffenden Frakturspaltes bei mechanisch stabiler Osteosynthese ist essenziell. Der Verbleib eines Frakturspaltes von mehr als $3 \mathrm{~mm}$ kann bei Anwendung einer stabilen Osteosynthese wie der Verplattung nicht zeitgerecht durch Knochenbildung mittels der sog. Spaltheilung überbrückt werden. Diese Situation führt quasi als Pseudarthrosenmodell mit hoher Sicherheit zu einer Heilungsverzögerung ( Abb. 2).

Alle Frakturen, die am Schaft nicht im Sinne einer primären Knochenheilung reponiert werden müssen sowie Frakturen mit Defektsituationen, werden nach dem Prinzip der Schienung fixiert. Die Schienung gewährleistet über induzierte Mikrobewegungen eine mechanische Stimulation der Frakturheilung und ermöglicht die Heilung der Fraktur über eine periostale Bildung von Frakturkallus. Eine solche Schienung kann extramedullär, z. B. über winkelstabile Plattenosteosynthesen, oder intramedullär über Marknagelosteosynthesen mit Verriegelung erfolgen. Auch bei geschienten Frakturen kann es durch zu große Mikrobewegungen zu einer Heilungsverzögerung kommen, die dann meist als hypertrophe Pseudarthrosen sichtbar werden. 

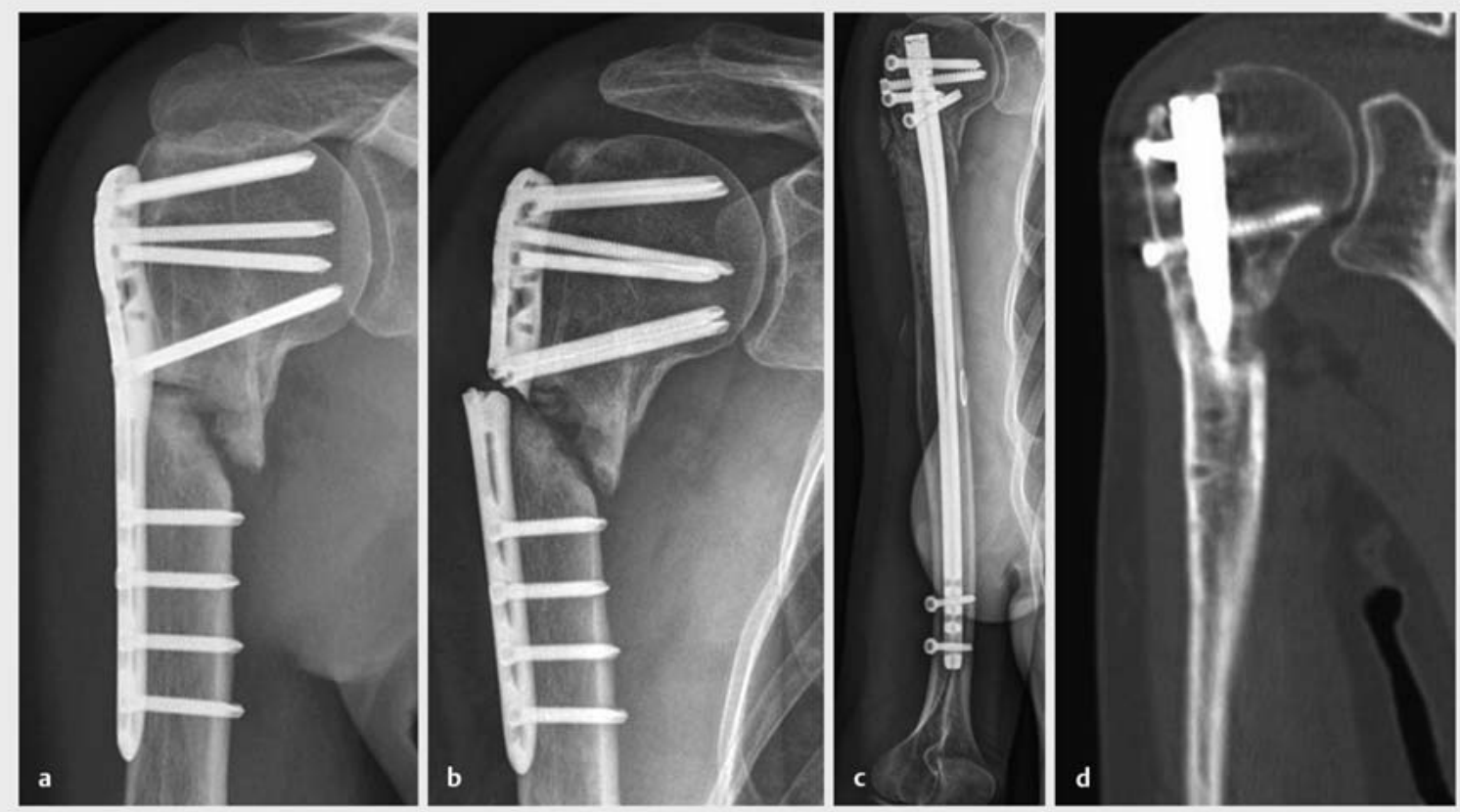

- Abb. 2 Versorgung einer proximalen Oberarmfraktur mit winkelstabiler Platte. a Reposition mit leichtem Varus und stärkerer Dehiszenz der Fraktur. b Bruch der Platte typischerweise in den besetzten Löchern auf Höhe der Fraktur. c Reosteosynthese mit proximalem Humerusnagel. d Fortschreitende Frakturheilung im CT.
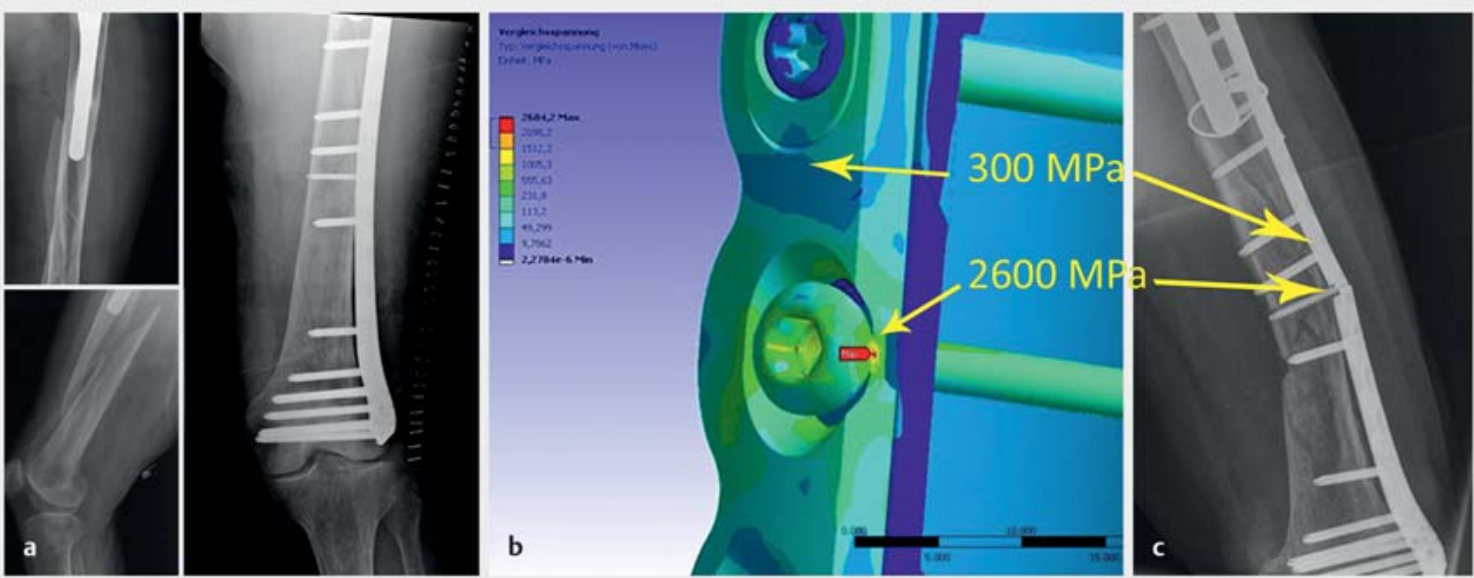

- Abb. 3 Periprothetische Fraktur am distalen Femur versorgt mit winkelstabiler Platte. a Die konventionelle Schraube, für die Adaptation der Platte an den Knochen verwendet, wurde belassen. b Mithilfe der Finiten Element Analyse können die Spannungen in der Platte berechnet werden. Die Spannung um die konventionelle Schraube ist neunmal höher als um die winkelstabile Schraube. c Durch Spannungskonzentration induzierter Bruch der Platte.

Um zu große Mikrobewegungen bei geschienten Osteosynthesen zu vermeiden, muss auf eine ausreichende Stabilität des Implantats geachtet werden. Winkelstabile Platten sollten dabei genügend Länge haben und mit mindestens 2, besser 3 Schrauben jeweils proximal und distal der Fraktur fixiert werden. Die frakturnahen Schrauben sollten zur Fraktur einen Mindestabstand von einem
Knochendurchmesser haben. Ungünstig sind Zugschrauben frakturnah mit hohem Anpressdruck, die eine Kerbspannung auf die Platte ausüben ( $\bullet$ Abb. 3). Bei sehr distalen Frakturen kann es durch die Hebelwirkung zu Instabilitäten kommen, die durch die Verwendung von Cerclagen oder additiven Platten verhindert werden können. 

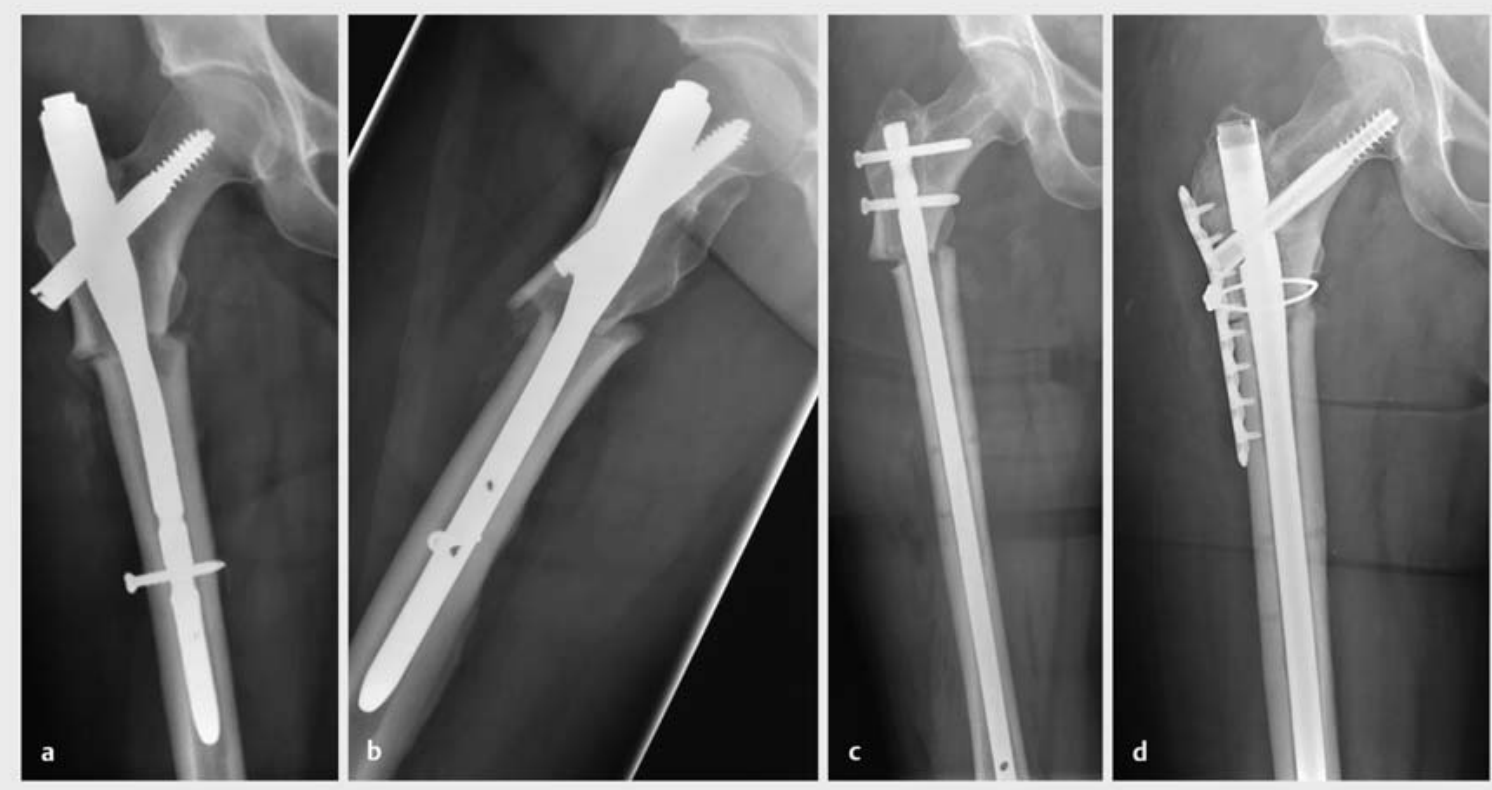

- Abb. 4 45-jährige Patientin mit instabiler subtrochantärer Fraktur. a Fraktur ist unzureichend mit Versatz und Varusstellung reponiert, der Nagel ist zu weit proximal platziert und die Schenkelhalsschraube zu kurz gewählt. b In der Seitprojektion ebenfalls unzureichende Reposition. c Revision mit geradem Marknagel, der bei identischem Einschlagpunkt die Varusfehlstellung verstärkt. d 2. Revision mit Reposition über Cerclage, korrektem Einschlagpunkt und stellungssichernder Antirotationsplatte.

Für die Stabilität der Marknagelosteosynthese ist sowohl die ausreichende Länge des Marknagels wie auch eine intramedulläre Stabilität durch Ausfüllung des Markraumes wichtig. Bei sehr proximal oder sehr distal gelegenen Frakturen muss durch Ausnutzung aller Verriegelungsoptionen des Nagels eine ausreichende Biegestabilität der Osteosynthese erreicht werden [9]. Die Schwachstelle der intramedullären Osteosynthese ist eine geringe Rotationsstabilität der Marknägel. Bei rotationsinstabilen Frakturen kann eine Heilungsverzögerung z. B. durch eine zusätzliche Plattenosteosynthese vermieden werden, die die Rotation der Hauptfragmente um den Nagel verhindert [10].

Essenziell für die Heilung der Schaftfrakturen ist die möglichst korrekte Wiederherstellung zunächst der Länge, insbesondere aber von Achse und Rotation. Die häufigen Achsabweichungen, z. B. am proximalen Femur in Varus, führen, sich gegenseitig befruchtend, zur Pseudarthrose und zum Implantatversagen. Dabei können primär korrekt reponierte Frakturen durch einen zu lateralen Einschlagpunkt eines Marknagels in eine Varusfehlstellung gebracht werden ( $\triangleright$ Abb. 4). Eine häufige Fehlstellung ist die Rotationsabweichung des Oberschenkels nach außen nach Marknagelung. Auch diese Fehlstellung stellt prinzipiell einen die Pseudarthrose prädisponierenden Faktor $\operatorname{dar}(\triangleright$ Abb. $\mathbf{5})$.

\section{Prävention}

Klassiker für die Pseudarthrosenbildung nach Osteosynthese langer Röhrenknochen lassen sich für Platten und Nägel definieren. Winkelstabile Platten müssen ausreichend lang schienen und über der Fraktur eine definierte Schwingungsstrecke aufweisen, um Plattenbrüche zu vermeiden. Marknägel sind ebenfalls in ausreichender Länge und markraumfüllend stabil einzubringen unter Vermeidung von Dehiszenz und Rotationsfehlern.

\section{Versagen von Osteosynthese- implantaten}

In aller Regel ist das Versagen von Osteosyntheseimplantaten ein Zeichen für eine verzögerte oder ausbleibende Frakturheilung. Osteosyntheseimplantate sind dafür spezifiziert, die mechanische Belastung über einen Zeitraum, der sich an der normalen Knochenheilung orientiert, unter Vollbelastung auszuhalten. Wenn die Belastung auf das Implantat im Verlauf der Heilungszeit nicht durch die fortschreitende Konsolidierung des Knochens sukzessive reduziert wird, kommt es durch die andauernde Belastung des Implantats zu einem Ermüdungsbruch. Dieser tritt meist im Bereich der Fraktur auf, weil dort die größten mechanischen Dehnungen bestehen ( $\bullet$ Abb. 6).

latrogene Ursachen für ein Implantatversagen entstehen durch Beschädigung der Oberfläche des Implantats im 

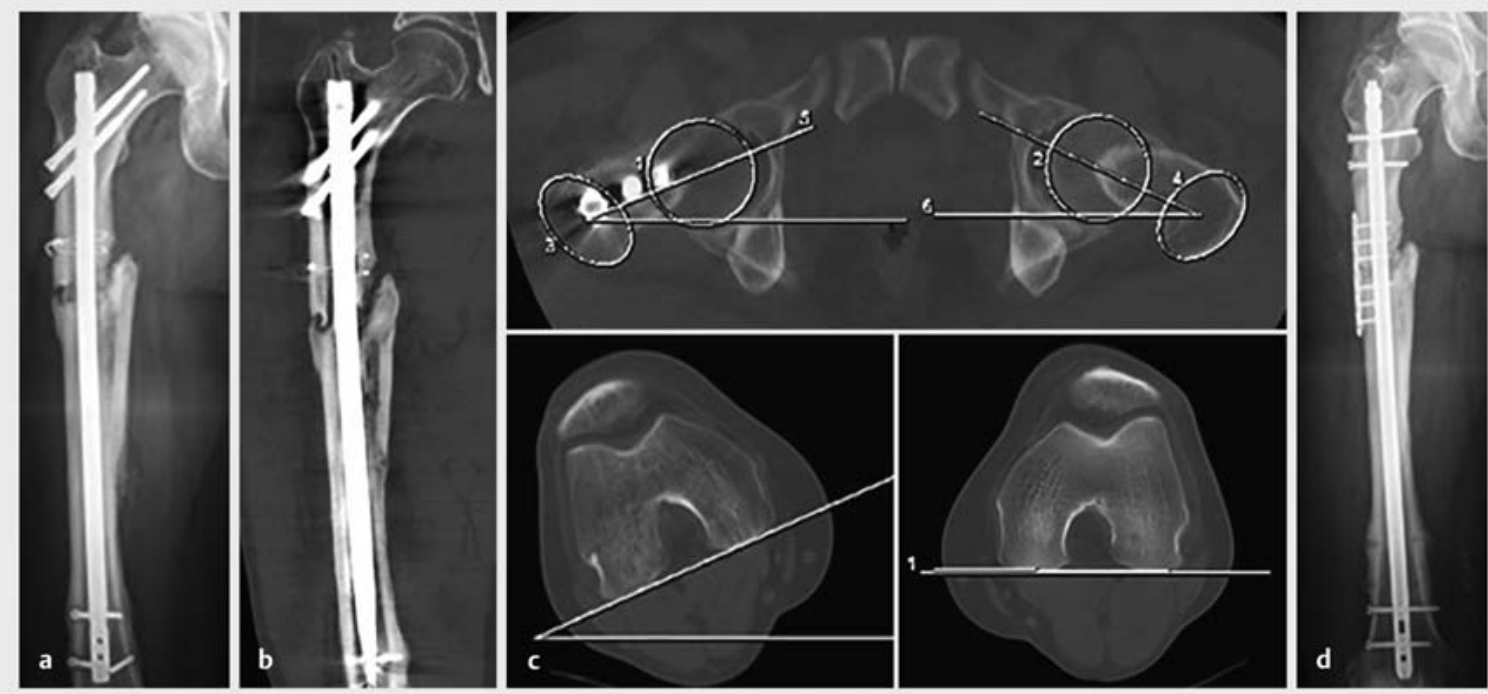

- Abb. 5 42-jähriger Mann, 8 Monate nach Erstversorgung. a Nagel zu kurz gewählt, distale Verriegelung gebrochen. b Hypertrophe Pseudarthrose im CT. c Außendrehfehler $30^{\circ}$. d Reosteosynthese mit Nagelwechsel, Derotation, Rotationssicherung über additive Platte.
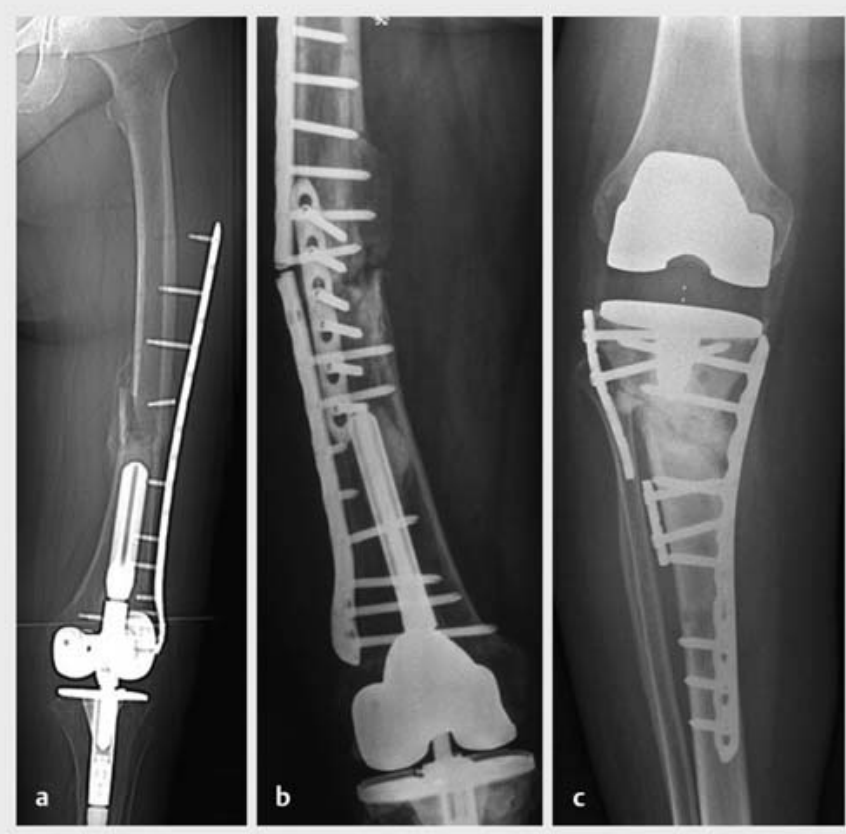

- Abb. 6 Problemzone periprothetische Frakturen. a Plattenausriss am Oberschenkelschaft, mögliche Ursache ist die teilweise nur monokortikale Schraubeneinbringung. b Plattenbruch bei nicht heilender Fraktur am Oberschenkel, Kritikpunkt ist die enge Schraubenbesetzung der winkelstabilen Platte ohne Schwingstrecke. c Plattenbruch an der proximalen Tibia, bei fehlendem Heilungspotenzial wäre ein Prothesenwechsel auf ein schaftgeführtes Design indiziert gewesen.
Verlauf der Operation. Riefen und Kratzer durch Bohrer oder Zange erzeugen Sollbruchstellen, an denen das Implantat unter zyklischer Wechsellast bricht. Daher müssen metallische Instrumentarien wie Bohrer oder Gewindeschneider immer mit besonderer Sorgfalt und unter dem Schutz von passenden Hülsen verwendet werden.

Eine prinzipiell als iatrogen verursacht anzusehende Ursache des Implantatversagens ist die falsche Wahl des Osteosynthesematerials ( $\boldsymbol{A}$ Abb. 7). Die Wahl des Implantats kann aus verschiedentlichsten Gründen, teils nachvollziehbar, teil unverständlich, im Widerspruch zur allgemeinen Meinung, zu Empfehlungen in Leitlinien oder Manualen, oder auch zu den Angaben des Herstellers stehen. Eine besondere Form einer außerhalb der Konvention stehenden Implantatwahl stellt die Off-Label-Indikation dar.

Nichtsdestotrotz sind Off-Label-Anwendungen nicht immer vermeidbar. Für sehr speziell gelagerte Fälle, wie z. B. sehr kleine oder sehr große Patienten, passen Standardimplantate nicht und müssen modifiziert oder gegen für andere anatomische Lokalisationen konzipierte Platten getauscht werden. Mit Blick auf die aktuelle Rechtslage empfiehlt es sich, die Verwendung gegenüber dem Patienten zu begründen und aufzuklären oder bei intraoperativer Entscheidungsfindung im OP-Bericht dezidiert aufzuführen. Während bis in die 90er-Jahre überwiegend universelle Plattensysteme (unterschieden wurden Großfragment- und Kleinfragment- sowie Fingerimplantate) zum Einsatz kamen, wandelte sich dies zunehmend und heute überwiegend hin zu Spezialimplantaten. Diese sind 

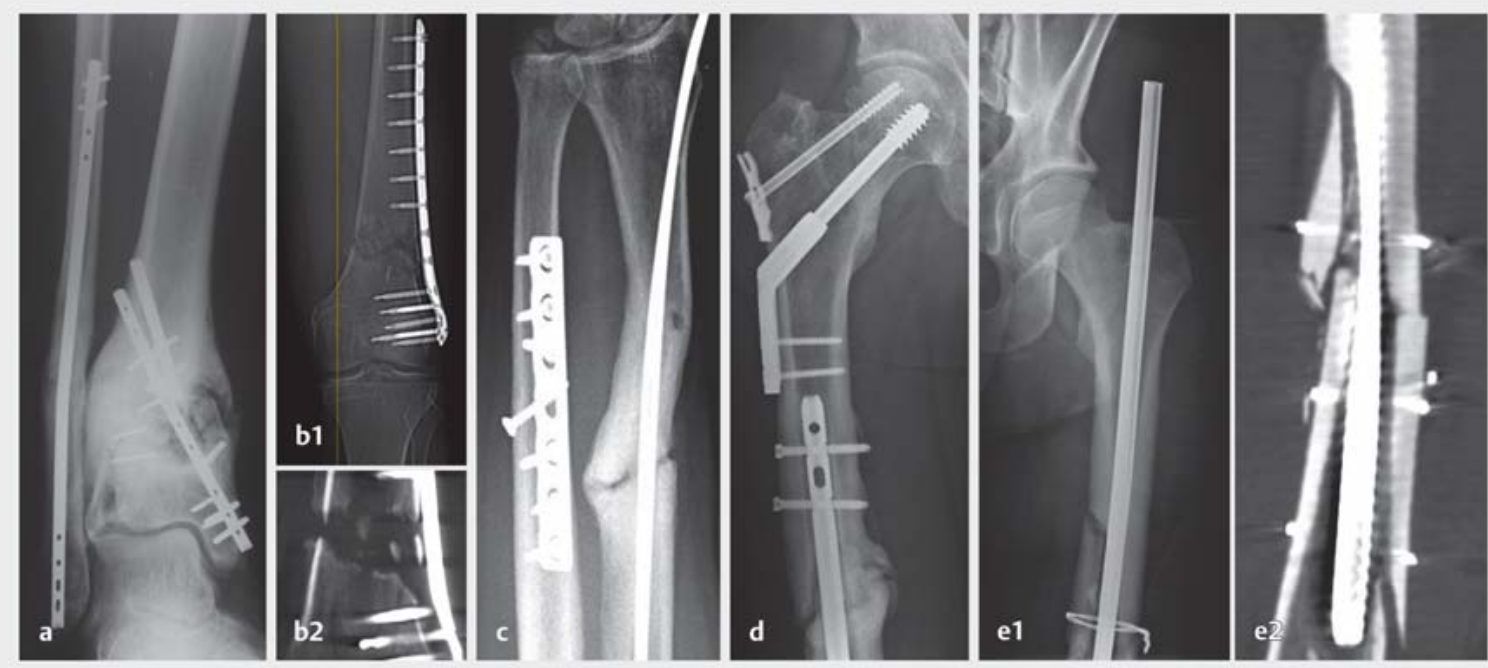

- Abb. 7 Implantatassoziierte Pseudarthrosen. a Ungeeignetes Implantat für eine Unterschenkelfraktur. b Fixierung auf Dehiszenz mit fraglich stabiler LISS-Platte (kurze monokortikale Schrauben). c Kombination Platte und Markdrahtung am Unterarm. d Zu kurzer Oberschenkelnagel bei Verlegung des Markraums proximal durch dynamische Hüftschraube wegen Schenkelhalsfraktur. e Wegen Auflaufens verklemmter und damit zu kurzer Oberschenkelmarknagel.
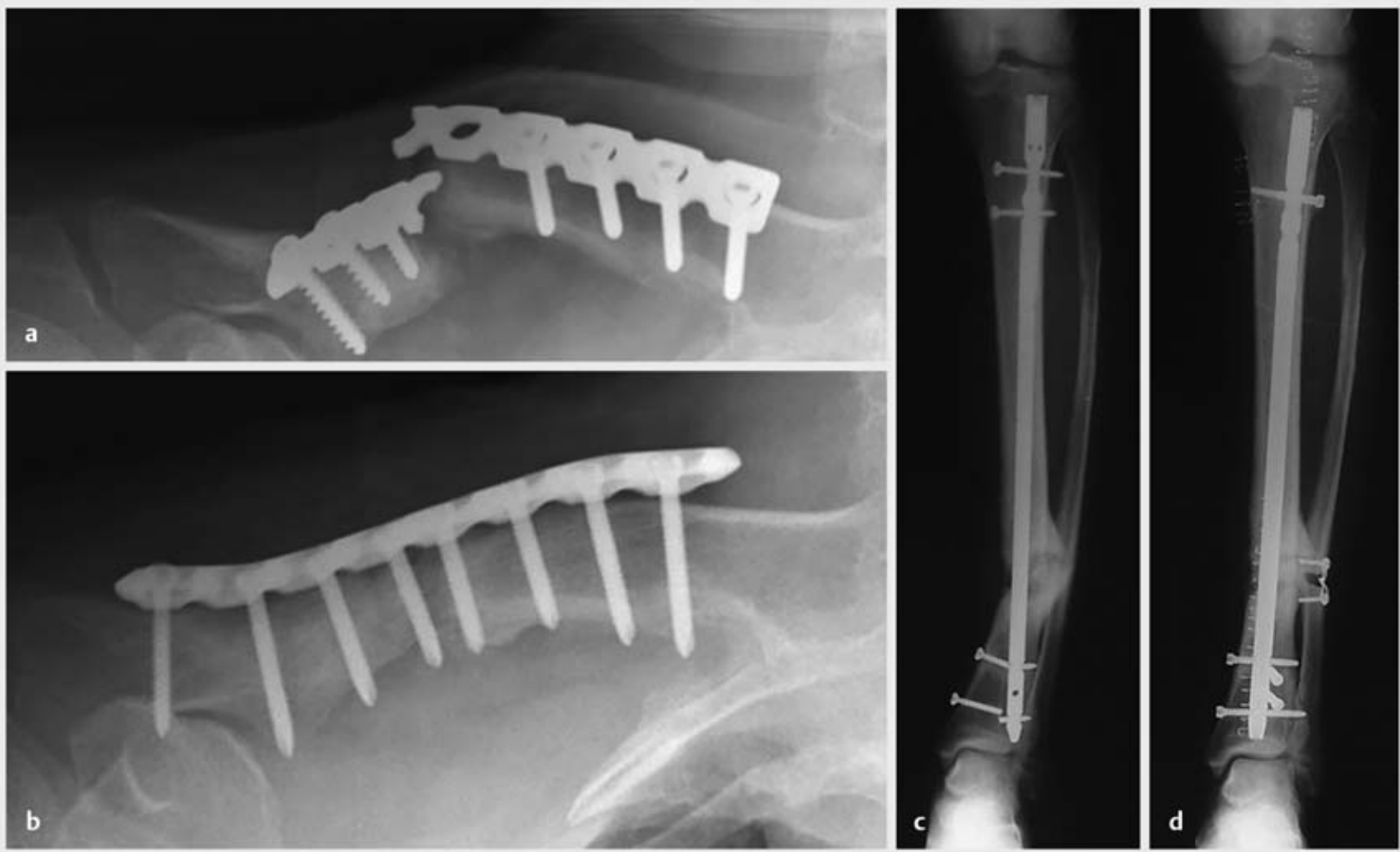

- Abb. 8 Implantate mit bekannter Neigung zum Versagen. a 3,5-mm-Rekonstruktionsplatte bei Klavikulafraktur, höhere Versagensraten sind zu beobachten. b Revision mit regulärer winkelstabiler Platte. c Unaufgebohrter Tibianagel bei Unterschenkelfraktur mit Trümmerzone, Bruch der distalen Verriegelungsschrauben bei „Selbstdynamisierung“. $\mathbf{d}$ Revision mit Achskorrektur und Austauschnagelung. 

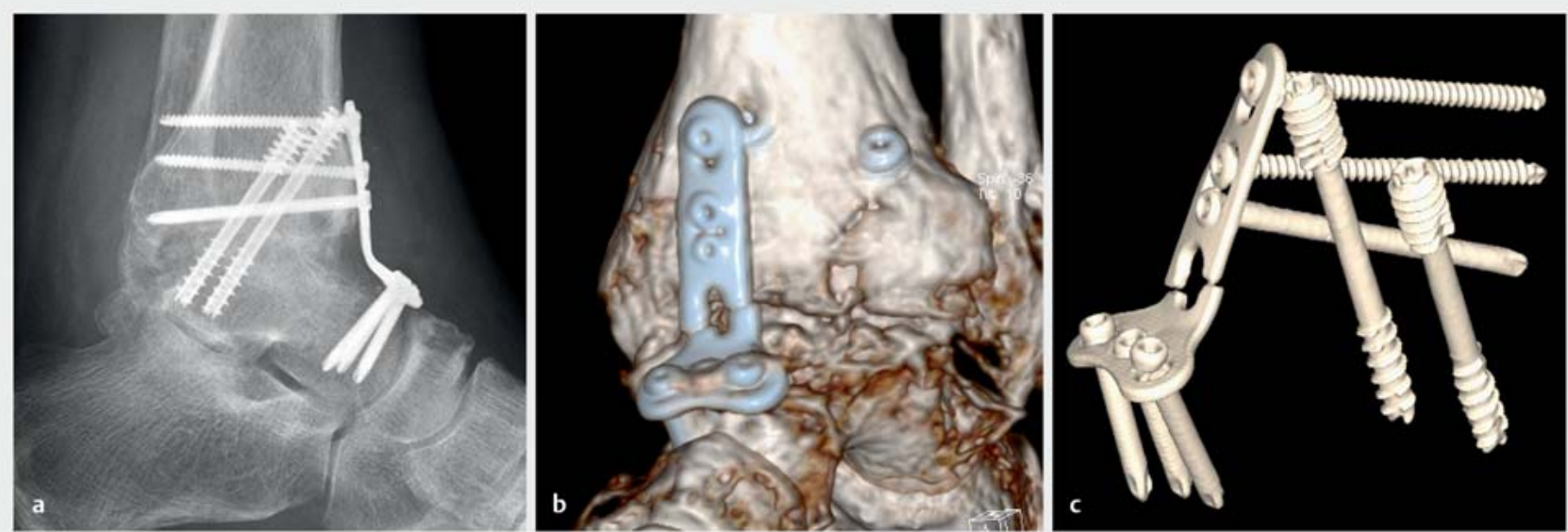

- Abb. 9 Implantatschwächung bei Off-Label-Anwendung. a Arthrodese des oberen Sprunggelenks mit direkter Verschraubung und ventraler Platte, Verwendung einer angebogenen winkelstabilen Radiusplatte. b Plattenbruch bei verzögerter knöcherner Konsolidierung. c Bruch im durch die Biegung zusätzlich geschwächten Längsloch der Platte.
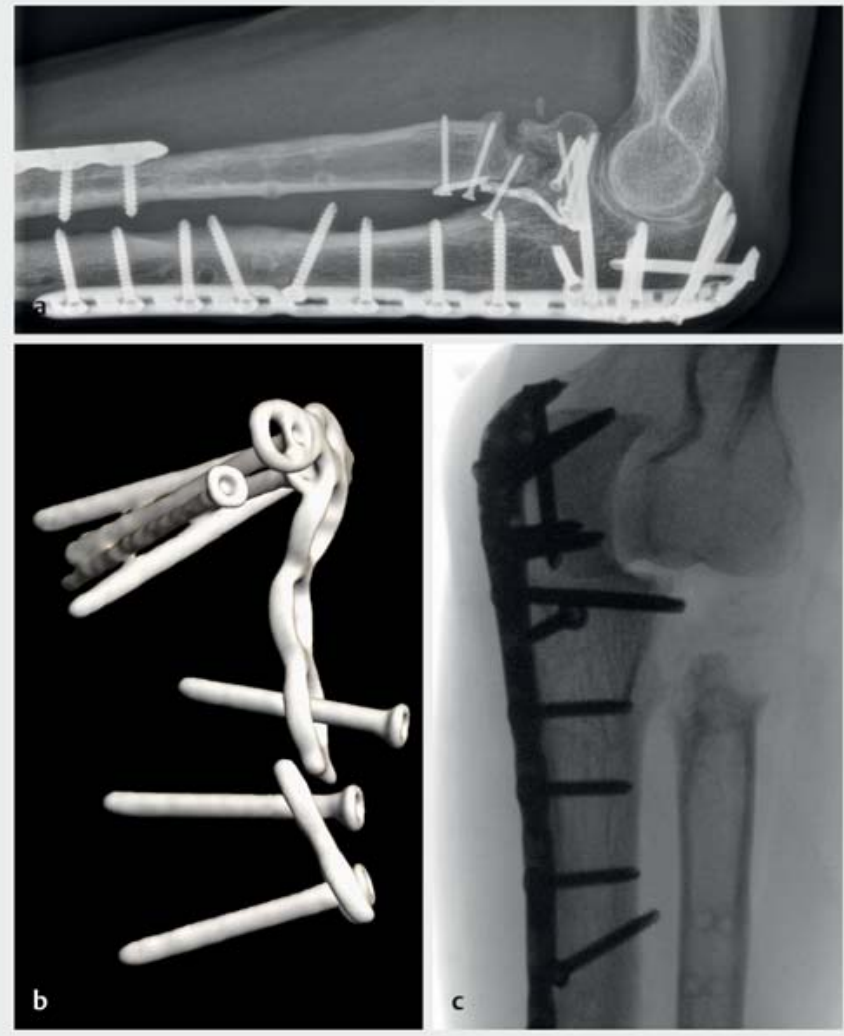

- Abb. 10 32-jähriger Mann mit komplexer Unterarmverletzung, 6 Monate nach Versorgung. a Winkelstabile Plattenosteosynthesen an proximaler Ulna und Radiusschaft, anatomische Platte für subkapitale Radiuskopffraktur. b Bruch der Platte im ehemaligen Frakturbereich bei Pseudarthrose. c Behandlung mit Metallentfernung und Resektion des Radiuskopfs. anatomisch für bestimmte Regionen konzipiert und werden häufig von den Herstellern zudem nur für bestimmte Indikationen empfohlen ( $\triangleright$ Abb. 8).

Anatomische Implantate haben den Vorteil, dass Anpassungen durch Biegung und Schränkung nicht mehr oder nur noch mit geringen Formveränderungen notwendig sind. Starke Verformungen führen zu Mikrofissuren des metallischen Implantats mit Entstehung einer Sollbruchstelle ( $\triangleright$ Abb. 9). Dies ist z. B. besonders ausgeprägt beim Zurückbiegen von Titanplatten.

Wesentlich häufiger als das Versagen des Implantatkonstruktes ist das Lockern und anschließende Auswandern von Schrauben aus der Platte oder dem Nagel. Selbst bei eigentlich winkelstabil eingebrachten Schrauben kann es durch die Biegewechselbelastung zu einem Lösen der Schrauben-Platte-Verbindung und zu einem Auswandern der Schraube kommen ( $\triangleright$ Abb. 10). Bei winkelstabilen Schrauben ist die Einhaltung der vorgegebenen Richtung durch Verwendung einer Bohrhülse zu sichern und zudem auf ein korrektes Anzugsmoment der Schraube zu achten [11].

\section{Prävention}

Implantatversagen mit Bruch oder Lockerung ist in aller Regel ein Zeichen einer verzögerten Knochenbruchheilung. Osteosyntheseimplantate sind nur für eine temporär begrenzte Stabilisierung designt und versagen bei ausbleibender Knochenbruchheilung in diesem Zeitfenster. Schwächungen des Implantatdesigns durch unbeabsichtigte Beschädigungen, wie z. B. Anbohren, sowie kalkulierte Schwächungen, wie Schränken und Biegen, sollten nach Möglichkeit vermieden werden. 


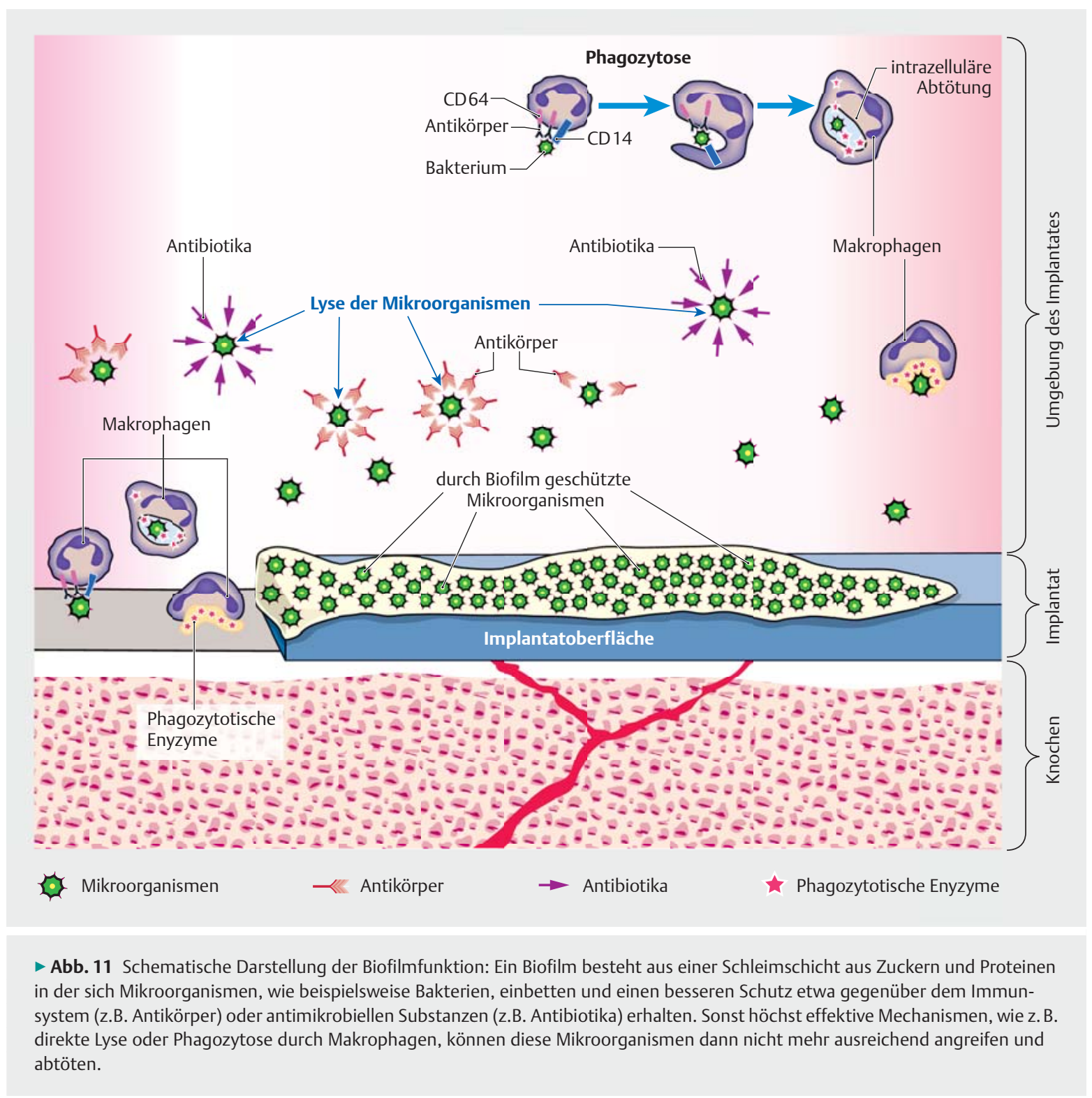

\section{Infekt und Pseudarthrose}

Neben der biomechanischen Instabilität und der biologischen Insuffizienz mit einer gestörten Durchblutungssituation im Bereich der Frakturzone kann auch eine lokalisierte bakterielle Besiedelung zur Beeinträchtigung eines zeitgerechten Frakturheilungsprozesses führen [12]. Neben dem Auftreten klassischer Entzündungszeichen mit einem klinisch meist sicher diagnostizierbaren floriden Infekt, treten auch sog. Low-Grade-Infekte auf. Diese meist unterschwellig verlaufenden Infektionen haben als einziges Symptom eine ausbleibende knöcherne Konsolidierung der Frakturzone. Unklar ist hierbei weiterhin die Häufigkeit einer solchen Low-Grade-Situation. So konnte unter Anwendung molekularbiologischer Verfahren gezeigt werden, dass bei 34 Patienten in $88 \%$ der Fälle Bakterien in der Pseudarthrosenzone nachweisbar waren [3].
Diese sog. Low-Grade-Infekte werden meist durch niedrig virulente Erreger, wie bspw. Staphylococcus epidermidis, hervorgerufen. Diese besitzen biofilmbildende Eigenschaften und können so von systemisch verabreichten Antibiotika nicht erreicht werden [13] ( $\bullet$ Abb. 11). Aktuell rücken insbesondere bei infektgefährdeten Situationen, wie offenen Frakturen, infektresistente Implantate in den Blickpunkt, die mit antibakteriellen Substanzen bzw. Materialien beschichtet sind. Ziel solcher Beschichtungen ist es, noch vor Ausbildung eines Biofilms die Beeinträchtigung des Frakturheilungsprozesses durch Bakterien zu minimieren. Für die Beschichtung können passive Oberflächenmodifikationen, welche die Adhäsion von Bakterien am Implantat minimeren sollen, von aktiven Oberflächenmodifikationen, die pharmakologisch aktive mikrobielle Substanzen wie Antibiotika, Antiseptika oder Metallionen freisetzen, unterschieden werden [14,15]. 


\section{Prävention}

Infektionsvermeidung hat höchste Priorität in der Frakturversorgung. Bei schweren und schwersten Weichteilschädigungen ist Stufenkonzepten mit primärer Fixateur-externe-Stabilisierung und sekundärer Definitivversorgung der Vorzug zu geben. Die Rolle infektresistenter Implantate ist für die Zukunft noch zu definieren.

\section{Prävention als Prinzip}

Mit Fokus auf die chirurgischerseits leistbare Vermeidung implantatassoziierter Pseudarthrosen lassen sich die Faktoren Planung, Übung und Kontrolle definieren. Eine sorgfältige und dokumentierte präoperative Planung minimiert Fehler der Implantatwahl und erhöht die Transparenz des Eingriffs für das ganze OP-Team. Digital gestützte Planungsprogramme ersetzen die früher üblichen mühsamen Skizzenzeichnungen und lassen sich im Kliniksystem digital dokumentieren.

Die Kompetenz auch schon am Beginn der chirurgischen Karriere hat durch das große Kursangebot für Osteosynthesetechniken eine solide und etablierte Grundlage erreicht. Kadaverworkshops mit z.T. präformierten Fraktursetzungen bilden eine sinnvolle Erweiterung mit Schulung der operativen Zugänge und einer Annäherung an die reale klinische Situation. Allerdings sind derartige Kursabsolvierungen vor dem Einsatz am Patienten bisher nicht systematisch eingefordert und auch nicht obligatorisch in die Facharztweiterbildung integriert.

Individuell sollte jede Osteosynthese intraoperativ über Bildwandler in 2 Ebenen und mit Lagekontrolle der gelenknahen Implantate dokumentiert und in der Nachmittagsbesprechung nach dem Mehrere-Augen-Prinzip validiert werden. Im weiteren Verlauf sind Röntgenkontrollen standardisiert und verbindlich zu definieren. Ziel ist die möglichst frühzeitige Erkennung eines Implantatversagens und einer drohenden Frakturheilungsstörung mit dann möglicher frühzeitiger Revision. Die Ausbildung einer klassischen Pseudarthrose wird unter diesen Maßnahmen eine Ausnahme darstellen. Für Dauerimplantate, wie z. B. Endoprothesen, haben sich umfassende Register auf regionaler, nationaler und internationaler Ebene bewährt. Eine entsprechende Entwicklung ist auch für die temporär wirkenden Osteosyntheseimplantate absehbar. Beschleunigen wird sich diese Entwicklung durch die europaweite Einführung der Medical Device Regulations (MDR), die eine studienmäßige Dokumentation der Langzeitverläufe auch von Osteosyntheseimplantaten zwingend erforderlich macht. Derartige systematische Register und Studienprotokolle haben erfahrungsgemäß das Potenzial, Unterschiede und Schwächen verschiedener Osteosyntheseformen in der Zukunft auch statistisch gesichert aufzuzeigen.

\section{Fazit}

Eine Mitwirkung des Implantats bei der Pseudarthrosenentstehung nach operativer Knochenbruchbehandlung muss in der großen Mehrzahl der Fälle diskutiert werden. Das Spektrum reicht von falschem Implantat für die gegebene Frakturform bis zur falschen Anwendung eines prinzipiell korrekten Implantates. Wechselwirkungen mit der Verletzungsschwere und der Patientencharakteristik sind praktisch immer vorhanden und erschweren die Analyse von Ursache und Wirkung. Für die Zukunft sind Verbesserungen durch obligatorische Schulungen der OP-Teams und durch systematische Erfassung der klinischen Verläufe zu erwarten.

\section{Autorinnen/Autoren}

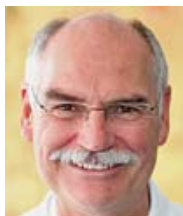

\section{Volker Bühren}

Prof. Dr. med., Institut für Biomechanik, Berufsgenossenschaftliche Unfallklinik Murnau und Paracelsus Medizinische Privatuniversität Salzburg

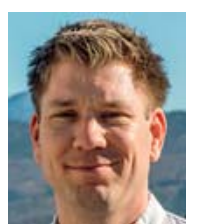

\section{Simon Hackl}

Dr. med., Facharzt für Orthopädie und Unfallchirurgie, Abteilung für Unfallchirurgie, Berufsgenossenschaftliche Unfallklinik Murnau

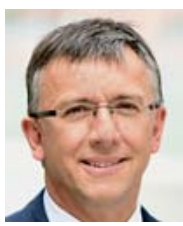

\section{Peter Augat}

Prof. Dr., Leiter Institut für Biomechanik, Berufsgenossenschaftliche Unfallklinik Murnau und Paracelsus Medizinische Privatuniversität Salzburg

Korrespondenzadresse

Prof. Dr. Peter Augat

Institut für Biomechanik

Berufsgenossenschaftliche Unfallklinik Murnau und

Paracelsus Medizinische Privatuniversität Salzburg

Prof.-Küntscher-Straße 8

82418 Murnau

Tel.: $08841 / 48-3058$

Fax: 08841/48-4573

biomechanik@bgu-murnau.de

\section{Literatur}

[1] Zimmermann, G. Verzögerte Knochenheilung. In: Biberthaler $P$, van Griesven M. Knochendefekte und Pseudarthrosen. Berlin: Springer; 2017: 63-75

[2] Steinhausen E. Low-Grade-Infekt: Muss jede Pseudarthrose biopsiert werden? Trauma Berufskrankh 2017; 19 (Suppl. 3): S267-S271

[3] Palmer MP, Altman DT, Altman GT et al. Can we trust intraoperative culture results in nonunion? J Orthop Trauma 2014; 28: $384-390$ 
[4] Giannoudis PV, Einhorn TA, Marsh D. Fracture healing: the diamond concept. Injury 2007; 38 (Suppl. 4): S3-S6

[5] Augat P, von Rüden C. Evolution of fracture treatment with bone plates. Injury 2018; 49 (Suppl. 1): S2-S7

[6] Augat P, Burger ], Schorlemmer S et al. Shear movement at the fracture site delays healing in a diaphyseal fracture model. J Orthop Res 2003; 21: 1011-1017

[7] Augat P, Bühren V. [Modern implant design for the osteosynthesis of osteoporotic bone fractures]. Orthopade 2010; 39: 397

[8] Baumgaertner MR, Curtin SL, Lindskog DM et al. The value of the tip-apex distance in predicting failure of fixation of peritrochanteric fractures of the hip. J Bone Joint Surg Am 1995; 77: 1058-1064

[9] Augat P, Bühren V. [Intramedullary nailing of the distal tibia. Does angular stable locking make a difference?]. Unfallchirurg 2015; 118: 311-317

[10] Eberle S, Gabel J, Hungerer S et al. Auxiliary locking plate improves fracture stability and healing in intertrochanteric fractures fixated by intramedullary nail. Clin Biomech 2012; 27: 1006-1010
[11] Sandriesser S, Rupp M, Greinwald M et al. Locking design affects the jamming of screws in locking plates. Injury 2018; 49 (Suppl. 1): S61-S65

[12] Calori GM, Albisetti W, Agus A et al. Risk factors contributing to fracture non-unions. Injury 2007; 38 (Suppl. 2): S11-S18

[13] Trampuz A, Zimmerli W. Diagnosis and treatment of infections associated with fracture-fixation devices. Injury 2006; 37 (Suppl. 2): S59-S66

[14] Alt V. Antimicrobial coated implants in trauma and orthopaedics - a clinical review and risk-benefit analysis. Injury 2017; 48: 599-607

[15] Romanò CL, Scarponi S, Gallazzi E et al. Antibacterial coating of implants in orthopaedics and trauma: a classification proposal in an evolving panorama. J Orthop Surg Res 2015; 10: 157

Bibliografie

DOI https://doi.org/10.1055/a-0902-3639

OP-JOURNAL 2019; 35: 241-251 @ Georg Thieme Verlag KG Stuttgart · New York ISSN 0178-1715 